\title{
Carboxy Terminus Heat Shock Protein 70 Interacting Protein Reduces Tau-Associated Degenerative Changes
}

\author{
Laiq-Jan Saidi $^{\mathrm{a}, \mathrm{b}}$, Manuela Polydoro ${ }^{\mathrm{a}}$, Kevin R. Kay ${ }^{\mathrm{a}}$, Laura Sanchez ${ }^{\mathrm{a}}$, Eva-Maria Mandelkow ${ }^{\mathrm{b}, \mathrm{c}, \mathrm{d}}$, \\ Bradley T. Hyman ${ }^{\mathrm{a}}$ and Tara L. Spires-Jones ${ }^{\mathrm{a}, \mathrm{e}, *}$ \\ ${ }^{a}$ Massachusetts General Hospital, Harvard Medical School, Charlestown, USA \\ ${ }^{\mathrm{b}}$ DZNE, German Center for Neurodegenerative Diseases, Bonn, Germany \\ ${ }^{\mathrm{c}}$ Caesar Research Center, Bonn, Germany \\ ${ }^{\mathrm{d}}$ Max-Planck-Institute for Metabolism Research, Cologne, Germany \\ ${ }^{\mathrm{e}}$ Centre for Cognitive and Neural Systems and the Euan MacDonald Centre, University of Edinburgh, UK
}

Handling Associate Editor: Alexis Stranahan

Accepted 1 October 2014

\begin{abstract}
One of the hallmarks of Alzheimer's disease is the formation of neurofibrillary tangles, intracellular aggregates of hyperphosphorylated, mislocalized tau protein, which are associated with neuronal loss. Changes in tau are known to impair cellular transport (including that of mitochondria) and are associated with cell death in cell culture and mouse models of tauopathy. Thus clearing pathological forms of tau from cells is a key therapeutic strategy. One critical modulator in the degradation and clearance of misfolded proteins is the co-chaperone CHIP (Carboxy terminus Hsp70 interacting Protein), which is known to play a role in refolding and clearance of hyperphosphorylated tau. Here, we tested the hypothesis that CHIP could ameliorate pathological changes associated with tau. We find that co-expressing CHIP with full-length tau, tau truncated at D421 mimicking caspase cleavage, or the short tau $\operatorname{RD}_{\mathrm{RD}} \Delta \mathrm{K} 280$ tau construct containing only the tau repeat domain with a tauopathy mutation, decreases tau protein levels in human $\mathrm{H} 4$ neuroglioma cells in a manner dependent on the Hsp70-binding TPR domain of CHIP. The observed reduction in tau levels by CHIP is associated with a decrease of tau phosphorylation and reduced levels of cleaved Caspase 3 indicating that CHIP plays an important role in preventing tau-induced pathological changes. Furthermore, tau-associated mitochondrial transport deficits are rescued by CHIP co-expression in $\mathrm{H} 4$ cells. Together, these data suggest that the co-chaperone CHIP can rescue the pathological effects of tau, and indicate that other diseases of protein misfolding and accumulation may also benefit from CHIP upregulation.
\end{abstract}

Keywords: Alzheimer's disease, caspase, CHIP, mitochondrial transport, tau protein

\section{INTRODUCTION}

The tau-protein is a natively soluble protein that plays a key role in the dynamics of microtubules.

\footnotetext{
*Correspondence to: Tara Spires-Jones, Centre for Cognitive and Neural Systems, School of Biomedical Sciences, College of Medicine and Veterinary Medicine, University of Edinburgh, 1 George Square, Edinburgh EH9 3EL, UK. Tel.: +44 1316511895; Fax: +44 1316511835; E-mail: Tara.spires-jones@ed.ac.uk.
}

In the pathological case of Alzheimer's disease (AD) tau becomes hyperphosphorylated, detaches from the microtubules, misfolds, and mislocalizes to the somatodendritic compartment where it aggregates into neurofibrillary tangles. These intracellular inclusions of abnormal fibrillar forms of tau play an important role in $\mathrm{AD}[1,2]$.

Pathological changes in tau damage cells at least in part by altering microtubule-dependent cellular 
transport. The overexpression of full-length tau in Chinese hamster ovary ( $\mathrm{CHO}$ ) cells [3], N2A cells [4], cultured retinal ganglion cells [4], NB2a/d1 cells [5] H4-cells [6], and primary cortical neuron cultures [6] led to an impairment of anterograde transport of a variety of kinesin cargos, including mitochondria. This leads to accumulation of mitochondria in the cell body where they cluster near the microtubule center [6]. Thies and Mandelkow [7] have shown previously that the co-expression of microtubule-associated protein/microtubule affinityregulating kinase 2 (MARK2) with tau can rescue these effects caused by tau overexpression in vitro. In confirmation of these in vitro studies, we have seen that pathological changes in a tau mouse model and human AD brain are associated with mitochondrial clustering in neuronal cell bodies [8]. Pathological tau changes are also postulated to be related to caspase activation. Caspases are activated before neurofibrillary tangles form in rTg4510 mice, and tau cleaved by Caspase 3 in the $\mathrm{C}$ terminus at $\mathrm{Asp}^{421}$ ( $\operatorname{tau} \Delta \mathrm{C}$ ) is found in new tangles [9].

The two major pathways for protein degradation in cells are through the ubiquitin-proteasome system and the autophagy-lysosome system [10, 11], both of which have been implicated in tau degradation in AD [12]. Misfolded tau proteins, like other natively unfolded molecules, can be detected and cleared by chaperone assisted mechanisms [13]. Recent data suggest that a critical mediator of refolding or clearance of hyperphosphorylated tau is via the HSP70/HSP90 heat shock pathways in which a specific E3 ubiquitin ligase, CHIP (carboxy terminus Hsp70 interacting protein), can recognize and target for degradation abnormal but not normal tau molecules [14-16]. CHIP is also implicated in regulation of Caspase 3 activity. In CHIP knockout mice, Caspase 3 activation is increased and caspase-cleaved tau levels are increased [17]. CHIP interacts more strongly with tau $\Delta \mathrm{C}$ than full-length tau [18], suggesting it is involved in caspase cleaved tau degradation. Here, we tested the hypothesis that overexpression of CHIP can reduce the levels of pathologically altered tau protein and prevent the associated caspase activation and mitochondrial transport deficits.

We find that co-expressing CHIP with tau in Human $\mathrm{H} 4$ neuroglioma cells decreases tau protein levels in a manner dependent on the HSP70-binding TPR domain of CHIP. CHIP also decreases tau phosphorylation and caspase activation, and rescues the mitochondrial clustering phenotype associated with tau overexpression. These data indicate that enhancing CHIP levels or func- tion is a promising therapeutic avenue for treatment of tauopathies.

\section{MATERIALS AND METHODS}

\section{Plasmid constructs}

In this study we overexpressed tau, CHIP, and GFP constructs in cell culture. Three tau constructs were used: the largest CNS tau isoform (441 residues, 2N4R, called 4RTau), the largest tau isoform lacking the last 20 residues mimicking caspase 3 cleavage of tau behind aspartate 421 (tauC3, 421 residues), and an aggregate prone tau construct ( $\operatorname{tau}_{\mathrm{RD}} \Delta \mathrm{K} 280$ or $\mathrm{K} 18 \Delta \mathrm{K} 280$, 129 residues) containing only the tau repeat domain $(4 \mathrm{R})$ with deletion of lysine at position 280, a mutation associated with frontotemporal dementia with Parkinsonism-17. The cloning of the 4R-tau construct and the cleaved caspase 3 4R-tau form (tauC3) were described previously [6]. Briefly the longest human tau isoform (4 repeat tau including exons 2 and 3) was carried out by standard PCR and ligation procedures. For 4R-tau using the primers 5'-CTCGAGATGGCTGAGCCCCGCCAGGAGTTCGAAG-3', containing an Xho-1 digestion site and 5'-AATAGGATCCAAACCCTGCTTGGCCAGGGA GGCAGAC-3' with a BamH1 digestion site. tauC3 was constructed by using the longest human tau isoform (4 repeat tau including exons 2 and 3) minus the last 60 bp with the primer $5^{\prime}$ TGCACCTCGAGACATGGCTGAGCCCCGCCAGG AGTTCGAAGTGCACCTCGAGACATGGCTGAGC CCCGCCAGGAGTTCGAAG- $3^{\prime}$ and $5^{\prime}$ TTGGATCC CTAGTCTACCATGTCGATGCTGCCG- $3^{\prime}$.

The PCR products were digested with Xho-1 and BamH1, along the pMAX-green fluorescent protein (GFP) vector (Amaxa, Walkersville, MD, USA) and ligated into the digested vector. The proaggregant tau construct tau $\mathrm{RD} \Delta \mathrm{K} 280$ harboring the FTDP-17 mutation K280 and tagged with GFP was described elsewhere [19].

For CHIP expression we used three previously described constructs: pcDNA-CHIP, pcDNA3CHIP $\Delta U$ lacking the U-box domain necessary for E3 ligase activity (residues 196-303 deleted) and pcDNA3-CHIP $\triangle$ TPR lacking the TPR domain necessary for Hsp70 interaction (residues 32-145 deleted) [22, 23]. Myc-tagged CHIP constructs were produced by subcloning CHIP, CHIP $\Delta \mathrm{U}$, and CHIP $\triangle$ TPR into pcDNA3.1-Myc/His (Invitrogen). GFP-expressing plasmid was used as a control. All vectors and constructs were verified by sequence analysis. 


\section{Cell culture}

Human H4 neuroglioma cells (HTB-148; ATCC) were cultured in Opti-Mem (Gibco, Carlsbad CA, USA) supplemented with $10 \%$ defined fetal bovine serum (FBS) and incubated at $37^{\circ} \mathrm{C}, 5 \% \mathrm{CO}_{2} . \mathrm{H} 4$ cells were plated $24 \mathrm{~h}$ prior to transfection, grown to 80-90\% confluency. Each dish was transfected with $4 \mu \mathrm{g}$ of the Vector containing CHIP or GFP and a GFP-tau construct with an equimolar ratio of DNA constructs using Superfect (Qiagen, Chatsworth, CA) according to the manufacturer's instructions.

\section{Toxicity assay}

To determine the levels of toxicity in our in vitro experiments, we collected $48 \mathrm{~h}$ after transfection the cell media of a 94-well plate and performed a ToxiLight $^{\mathrm{TM}}$ assay (Lonza), according to the manufacturer's protocol. ToxiLight ${ }^{\mathrm{TM}}$ is a non-destructive cytotoxicity assay kit that measures the release of adenylate kinase from damaged cells into the culture medium.

\section{Pulse-chase experiment}

To confirm that the effects of CHIP were on degradation instead of decreased translation, $\mathrm{H} 4$ cells were plated in 6-well dishes and transfected with $2 \mu \mathrm{g}$ of the vector containing CHIP or GFP and a GFP-tau construct as described above. After $40 \mathrm{~h}$ transfection, the media (Opti-Mem+10\% FBS) was changed into H4-media containing $5 \mu \mathrm{M}$ of cyclohexamide (SigmaAldrich) that blocks new protein translation. The drug was reconstituted with 100\% DMSO (Sigma) and prepared in sterile PBS. Cells were cultured for another $8 \mathrm{~h}$ and scraped into lysis buffer.

\section{Western blot analysis}

For western blot analysis, H4 cells were harvested $48 \mathrm{~h}$ after transfection. After washing the cells once with ice-cold PBS, and scraping into complete Lysis-M-EDTA-free lysis buffer (Roche), containing complete protease inhibitors cocktail (Roche), the cells were allowed to lyse for $30 \mathrm{~min}$ on ice. Cell lysates were centrifuged at $10,000 \mathrm{rpm} 9,600 \times \mathrm{g}$ for $10 \mathrm{~min}$ at $4{ }^{\circ} \mathrm{C}$. Protein concentration was determined using a BCA-Assay (Thermo Scientific) according to the manufacturer's protocol. Total protein lysates $(20 \mu \mathrm{g}$ of total protein) were solubilized in lithium dodecyl sulfate (LDS) sample buffer under reducing agent. As a loading marker the pre-stained standard SeeBlue ${ }^{\circledR}$
Plus 2 (Life Technologies-Invitrogen) was used. Total proteins were separated by gel-electrophoresis using 10-20\% Tris/glycine gels (Invitrogen) and transferred onto PVDF (Millipore) in transfer buffer containing $20 \%$ methanol and blocked in blocking buffer $(5 \%$ Milk in TBST) for $1 \mathrm{~h}$ at room temperature, followed by incubation with primary antibodies. The following antibodies and dilutions were used: $\beta$-actin (Sigma; $1: 5,000)$, total tau (DAKO; $1: 10.000$ ), human tau, HT7 (Thermo Scientific, Rockfort IL, USA; 1 : 5,000), caspase cleaved tauD421, tauC3 (Invitrogen Corporation, Camarillo CA, USA; 1:500), phosphotau antibody 12E8 (gift from P. Seubert, Elan Pharma; $1: 5,000)$, myc (Abcam; $1: 2,000$ ), total caspase 3 (Cell Signaling; 1:500), cleaved caspase 3 (Cell Signaling; $1: 500$ ), and HSP 70 (StressMarq Biosciences Inc., Victoria, Canada). Immunoreactivity was visualized using Hyperfim ${ }^{\mathrm{TM}}$ ECL (GE HealthcareLimited, Backinghamshire, UK) and exposure on x-ray films. Densitometric and molecular weight analyses were performing using Image $\mathbf{J}$ software (National Institutes of Health). Band density values were normalized to $\beta$ actin, or to total tau levels when tau phosphorylation levels were analyzed or to total caspase 3 when tau level cleaved caspase 3 were analyzed.

\section{Immunocytochemistry and calculation of mitochondrial distribution}

$\mathrm{H} 4$ cells were co-transfected with one of the tau constructs described above and either CHIP or GFP (as a control). $24 \mathrm{~h}$ after transfection, mitochondria were labeled with Mitotracker Red (Invitrogen) for $30 \mathrm{~min}$ under normal growth conditions. Cells were then fixed in $4 \%$ paraformaldehyde for $20 \mathrm{~min}$ and permeabilized for $15 \mathrm{~min}$ with $0.5 \%$ Triton $\mathrm{X}-100$ at room temperature. Immunocytochemistry was performed using primary antibodies to detect human tau (HT7, Thermo Scientific, Rockford, IL, USA, mouse, 1 : 1000; polyclonal total tau antibody, DAKO, rabbit, $1: 5000$ ), and the myc tagged CHIP, mouse $1: 200$ : rabbit anti-myc tag, Upstate (Millipore) 1:200). The following fluorescently labeled secondary antibodies were used: goat-anti-mouse Alexa-Fluor $488(1: 500)$ and goatanti-rabbit Alexa-Fluor 488 (1:500, both were used from Invitrogen, Eugene, OR), goat-anti-mouse Cy5 (Thermo Scientific, $1: 200$ ) and goat-anti-rabbit Cy5 (Jackson ImmunoResearch, Westgrove PA, 1:200). Cells were observed using a Leica DMRE confocal microscope (Wetzler, Germany) and a $63 \times 1.4$ numerical aperture plan apochromatic oil objective. 
To analyze mitochondrial distribution in the cells, images from each condition ( $n=60 \mathrm{H} 4$ cells per transfection condition) were acquired and analyzed using Image J software as described previously [6]. Briefly, the green (for GFP constructs) and red channels were thresholded separately (to the edge of the intensity histogram). The cell body was outlined in the GFP channel and the analyze particles function in Image $J$ was used on the red channel to find the percentage area occupied by mitochondria.

\section{Statistics}

Data are presented as mean \pm SEM. Statistical analysis were done using GraphPad Prism5 (GraphPad, California, US) and JMP software. Normality of data was assessed with Shapiro-Wilks tests. Normally distributed data (western blot quantifications) were analyzed with parametric statistics. Groups were compared using two tailed, unpaired Student's test or One-Way ANOVA with a Bonferroni's post hoc correction. Statistical significance is indicated as follows: ${ }^{*} p<0.05,{ }^{* *} p<0.001,{ }^{* * *} p<0.0001$.

Mitochondrial distribution data were not normally distributed so are presented as box plots, which display the median value (line inside the box), upper quartile (top of the box), lower quartile (bottom of the box), 90th percentile (top whisker), 10th percentile (bottom whisker), and all values bellow the 10th and above the 90th percentile (potential outliers) shown as dots. Nonparametric statistics (Kruskal-Wallis tests) were used to analyze these data.

\section{RESULTS}

\section{CHIP decreases the amount of total-tau in a TPR-domain dependent manner}

To determine whether CHIP degrades several pathological forms of tau in vitro, $\mathrm{H} 4$ cells were transfected with tau and either CHIP or GFP (as a transfection control) for $48 \mathrm{~h}$. Densitometry analysis of total tau levels of H4- cell lysates on SDS-PAGE showed a 2.0 to 2.5 fold lower levels of tau (4R-tau, tauC3, and $\mathrm{K} 18 \Delta \mathrm{K} 280$ ) with co-expression with CHIP, respectively (Fig. 1). Toxicity assays revealed that neither CHIP nor any of the tau constructs caused cell death compared to the control GFP vector (Supplementary Fig. 1). Probing for phospho-tau with the 12E8 antibody in cell lysates from K18 $\Delta \mathrm{K} 280$ transfected cells reveals that tau phosphorylation is also decreased by CHIP overexpression (Fig. 1).
Several publications suggest that molecular chaperones, e.g., heat shock protein 70 and 90 (Hsp70, Hsp90) play a fundamental role in the clearance of misfolded proteins including tau [12]. The co-chaperone CHIP interacts with Hsp70/Hsp90 to bind unfolded and misfolded proteins. Co-transfection of CHIP with 4R-tau leads to a significant increase in Hsp70 levels, however this is not the case with wither tauC3 or K18DK280 constructs (Supplementary Fig. 2) suggesting that increased HSP70 protein levels are not necessary for the observed reduction in tau levels. Despite the lack of clear increases in HSP70 levels, the effects of CHIP on tau levels could be due to CHIP binding HSP70. CHIP has two important domains for his chaperone activity: the amino terminus tetratricopeptide repeat (TPR) domain that links CHIP to Hsp70 and Hsp90 [24] and the carboxyl terminus containing the U-box that is important for the E3 ubiquitin ligase function of CHIP. Either of these domains could be critical for degradation of tau since ubiquitination targets proteins for degradation and CHIP is known to interact with full-length tau in a complex with $\mathrm{Hsc} 70 / \mathrm{Hsp} 70$ and poly-ubiquitinated tau [17]. To decipher the mechanism by which CHIP mediates tau degradation, we co-transfected deletion mutant constructs of CHIP lacking the U-box or TPR domain (CHIP $\triangle \mathrm{U}$ and CHIP $\triangle \mathrm{TPR}$ ) with tau and the total tau level was analyzed $48 \mathrm{~h}$ after transfection. Interestingly, a significant decrease in tau levels was seen with the co-transfection of CHIP $\Delta \mathrm{U}$ (U-box deleted) when transfected with either K18 $\Delta \mathrm{K} 280$ or 4R-Tau. However, transient co-expression of CHIP $\triangle \mathrm{TPR}$ (containing the U-box domain) did not lead to any change in total tau levels, compared to cells transfected with tau and no CHIP (Fig. 2). Because the TPR domain of CHIP is essential for Hsp70 interactions, these data suggest that the CHIP-mediated reduction of tau depends on Hsp70 interactions.

To confirm that the chaperone-mediated decrease of tau-associated degenerative changes was dependent on protein degradation and not transcriptional downregulation, we performed a pulse-chase experiment. $40 \mathrm{~h}$ after co-transfection of tau (K18 $\Delta \mathrm{K} 280)$ with CHIP or control vector, cells were treated with $5 \mu \mathrm{M}$ cyclohexamide or DMSO. Cells were harvested $8 \mathrm{~h}$ after cylohexamide treatment and the level of total tau was assessed via western blot analysis. After cyclohexamide treatment, we could still detect a significant decrease in tau levels in cells that were co-transfected with CHIP indicating that the reduction in tau levels due to CHIP is due to degradation and not to transcriptional down-regulation (Supplementary Fig. 3). 


\section{A}

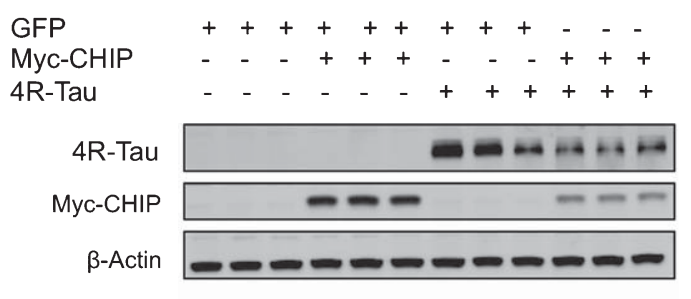

B

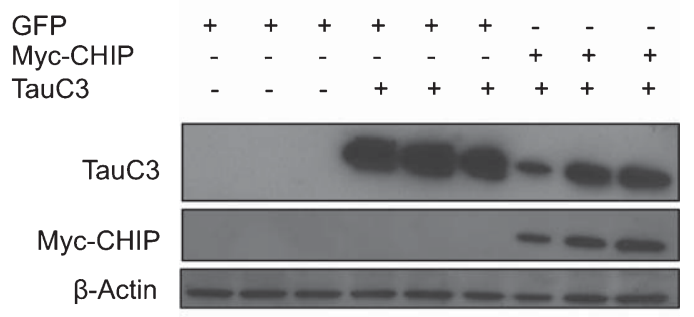

\section{C}

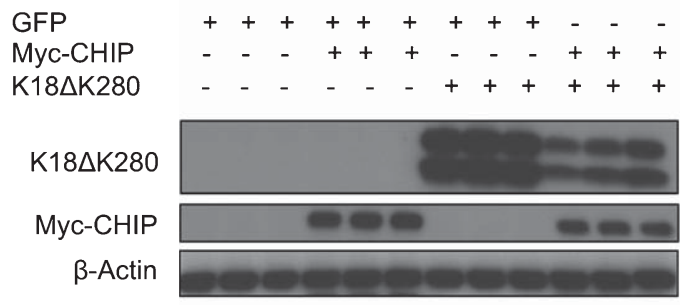

\section{D}

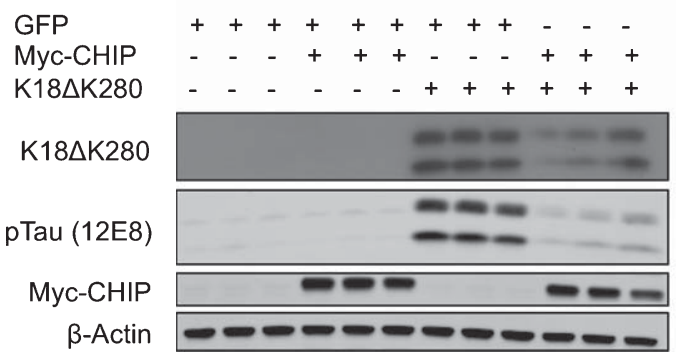

$64 \mathrm{kDa}$

$35 \mathrm{kDa}$

$42 \mathrm{kDa}$

$50 \mathrm{kDa}$

$35 \mathrm{kDa}$

$42 \mathrm{kDa}$

$14 \mathrm{kDa}$

$35 \mathrm{kDa}$

$42 \mathrm{kDa}$

$14 \mathrm{kDa}$

$14 \mathrm{kDa}$

$35 \mathrm{kDa}$

$42 \mathrm{kDa}$
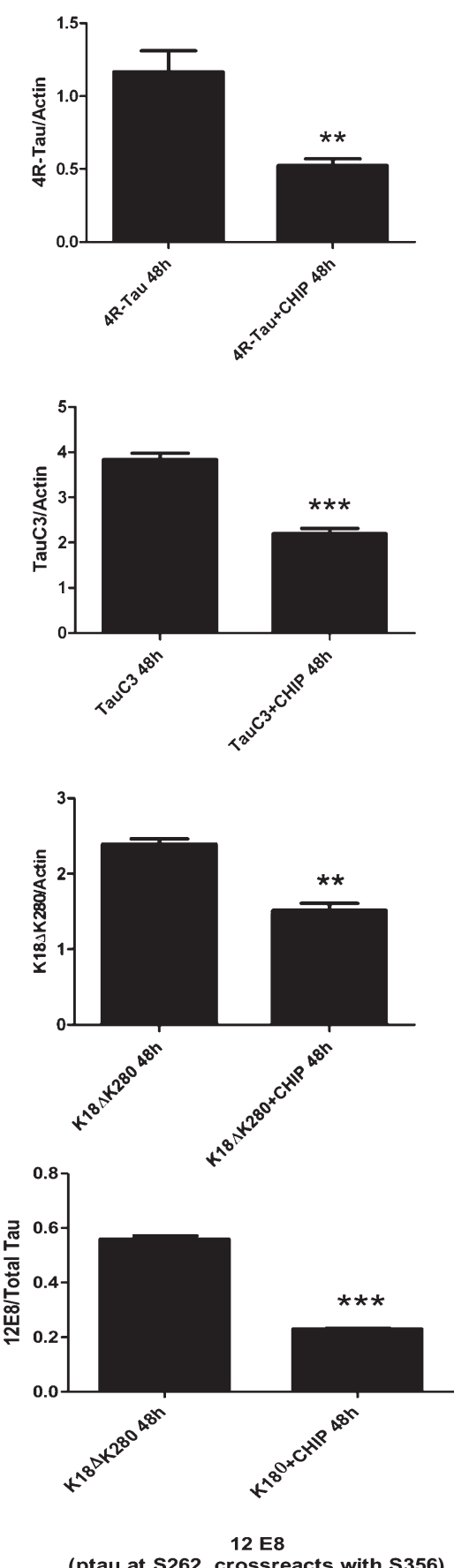

(ptau at S262, crossreacts with \$356)

Fig. 1. CHIP co-expression reduces levels of tau and phosphorylated tau. H4-cells were co-transfected with 4R-Tau (A), TauC3 (B), or K18 $\triangle$ K280 (C, D) and either CHIP or control vector GFP. Total cell lysates were analyzed for total-Tau (HT7) and CHIP (myc-Ab), revealing a significant reduction in tau protein levels with chip co-expression with all 3 tau constructs (A-C). To assess tau phosphorylation, lysates from cells transfected with K18 $\Delta 280$ and CHIP or GFP were analyzed for total tau (K9JA), phospho-tau at S262 (12E8) and CHIP (myc-Ab), revealing a reduction in tau phosphorylation with CHIP (D). Band densities were quantified using ImageJ and normalized to actin (A-C) or total tau (D) as a loading control. Data are presented as means \pm S.E.M. 
A

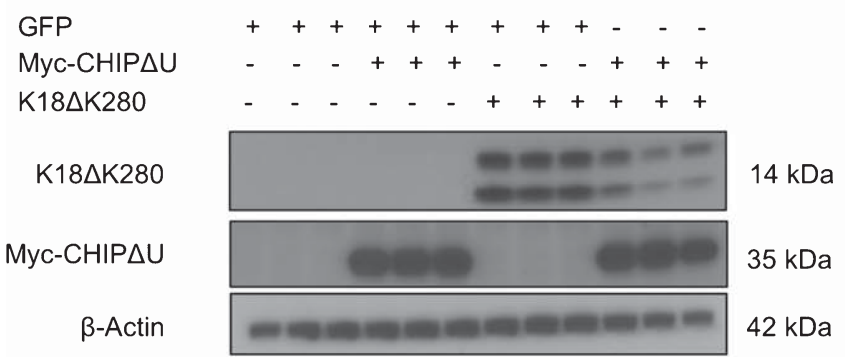

B
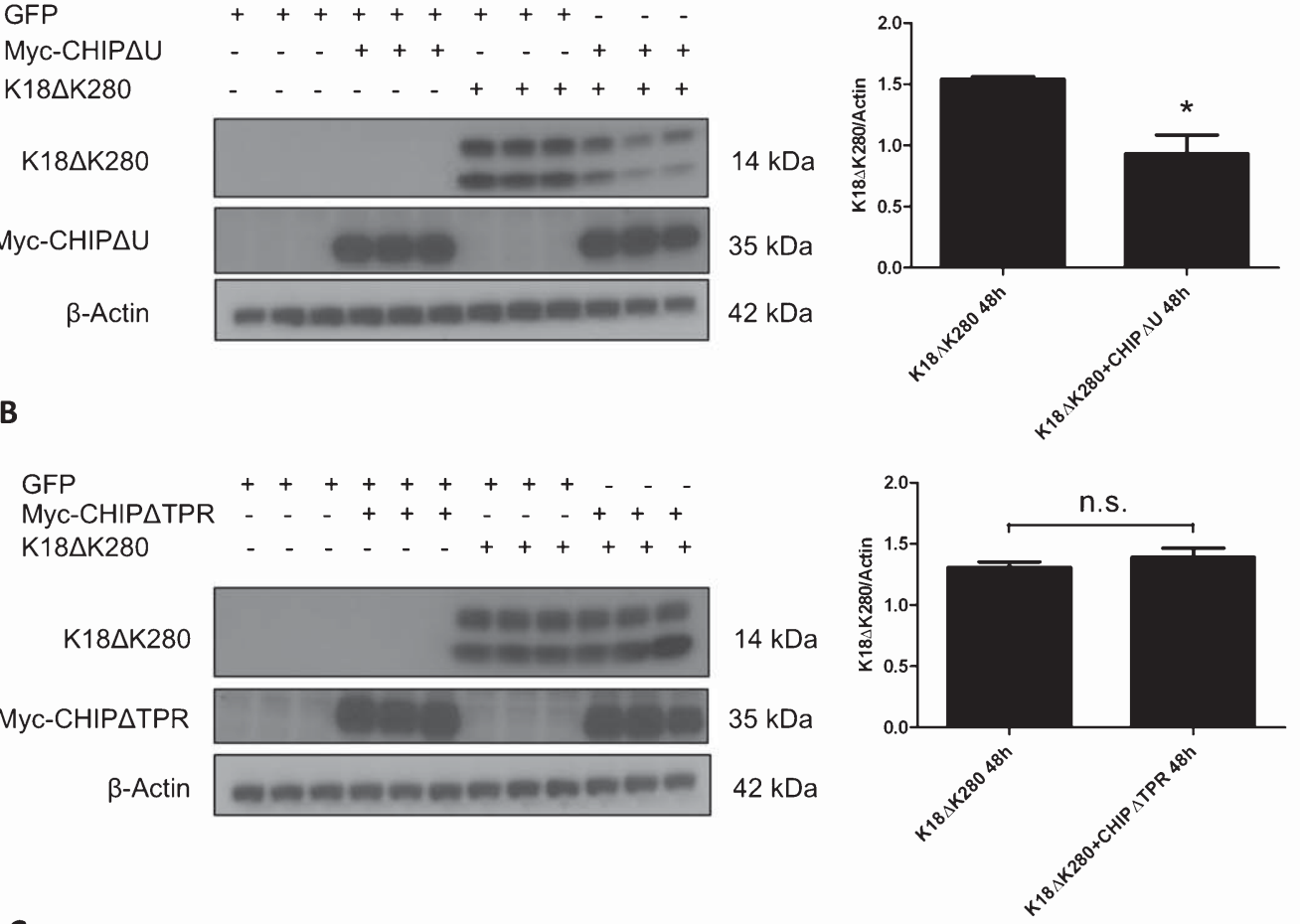

C

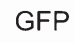

Myc-CHIP $\triangle U$

$+++++++++-\ldots$

4R-Tau

4R-Tau

- $-\quad-\quad-\quad+++++$

(R-Taun

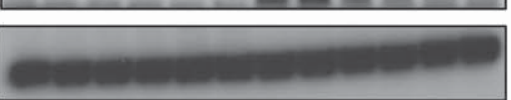

$64 \mathrm{kDa}$

$\beta$-Actin

$42 \mathrm{kDa}$

D

$\mathrm{GFP}+++++++++-t_{-}+$

4R-Tau $\quad-\quad-\quad-\quad-\quad-\quad-\quad++++++$

4R-Tau

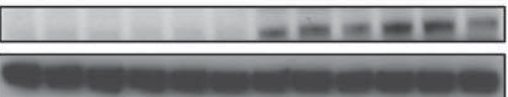

$64 \mathrm{kDa}$

$\beta$-Actin

$42 \mathrm{kDa}$

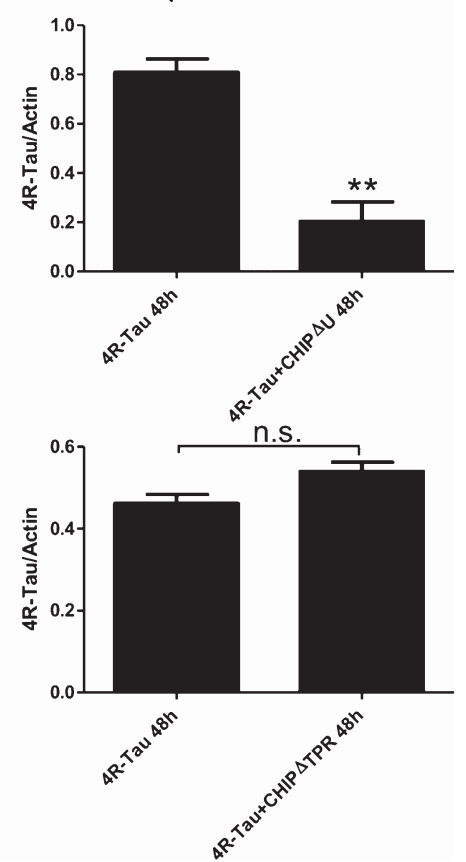

Fig. 2. The TPR-domain of CHIP is necessary for reduction in tau levels. H4-cells were co-transfected for $48 \mathrm{~h}$ with K18 $\Delta \mathrm{K} 280$ (A,B), $4 \mathrm{Rtau}$ (C,D), and either control vector (GFP), CHIP lacking the U-box domain (CHIP $\triangle$ U A,C) or CHIP lacking the TPR domain (CHIP $\triangle$ TPR B,D). Total cell lysates were analyzed for total Tau level (using K9JA) and CHIP $\Delta$ U/CHIP $\Delta$ TPR (myc-Ab). Band densities were quantified using ImageJ, normalized to actin as a loading control. Data are presented as means \pm S.E.M. 
A
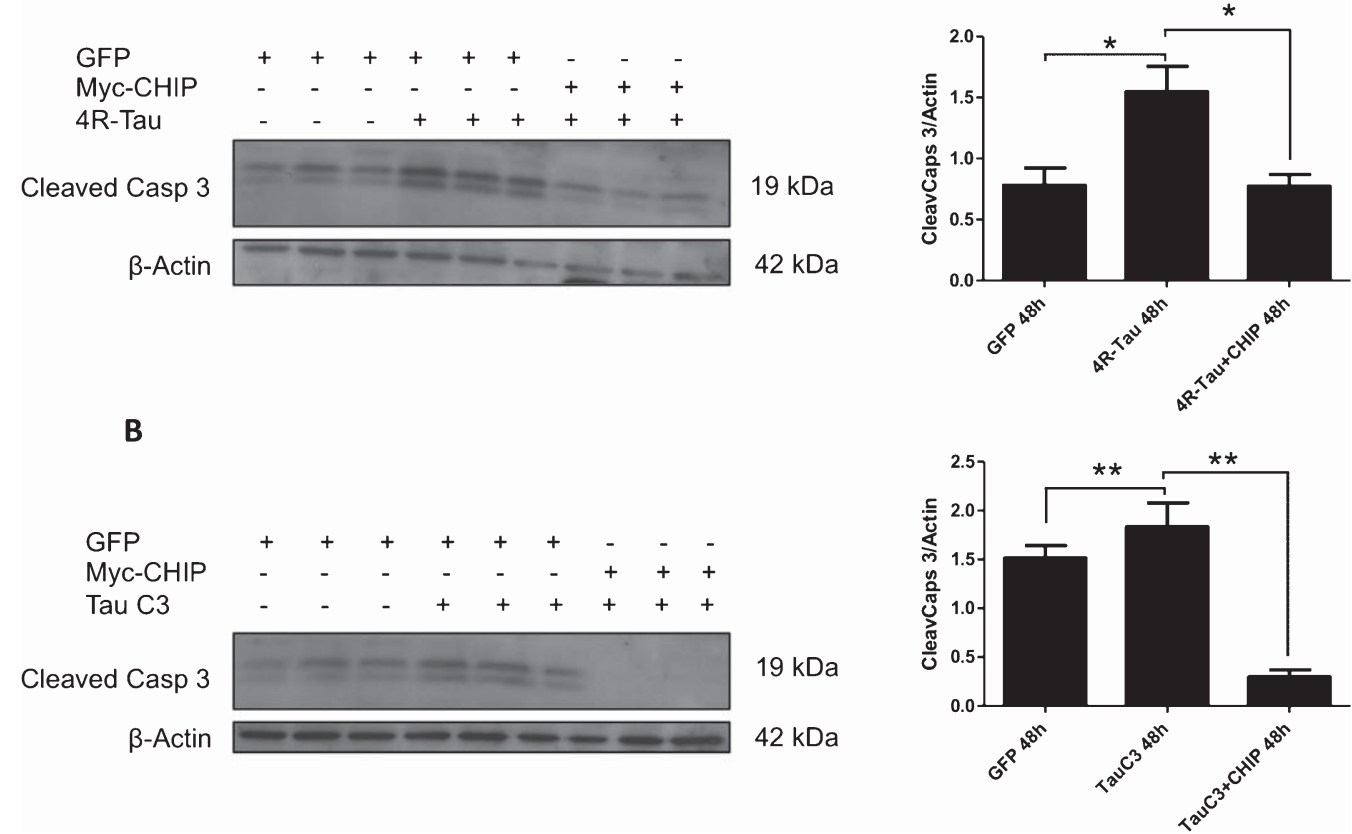

Fig. 3. CHIP reduces tau-induced caspase 3 activation after $48 \mathrm{~h}$. H4-cells were co-transfected for $48 \mathrm{~h}$ with $4 \mathrm{R}$-tau (A) or TauC3 (B), and either control vector (GFP) or CHIP. Total cell lysates were analyzed for cleaved caspase 3 levels and actin levels. Band densities were quantified using ImageJ, normalized to actin. Data are presented as means \pm S.E.M.

A

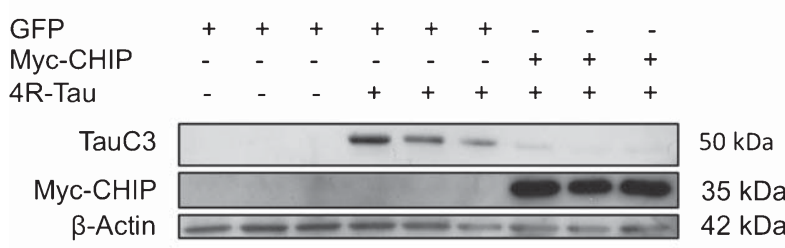

B

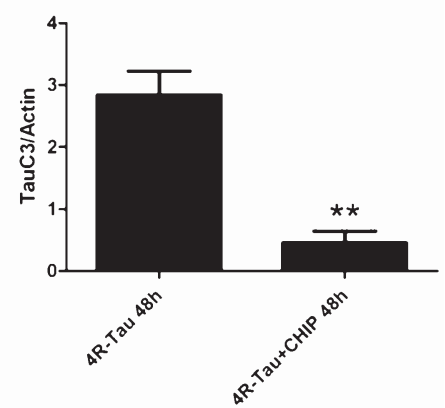

Fig. 4. CHIP decreases an abnormal truncated form of tau (TauC3) after 4R-Tau expression for $48 \mathrm{~h}$. H4-cells were co-transfected for $48 \mathrm{~h}$ with 4R-Tau and either control vector (GFP and CHIP). Total cell lysates were analyzed for total tau level (tauc3 specific antibody) and CHIP (myc-Ab) (A). Band densities were quantified using ImageJ, normalized to actin as a loading control (B). Data are presented as means \pm S.E.M.

\section{CHIP decreases active caspase-3 and caspase cleaved tau levels}

Recent data suggest that caspases are involved in the accumulation of tau pathology [10, 25, 26], and reductions in CHIP have been shown to cause caspase activation and increased caspase-cleaved tau levels [19]. Here we test whether CHIP overexpression can lead to a reduction of cleaved caspase-3. Tau overexpression increased active caspase 3 levels, and co-expression of CHIP reduced cleaved caspase 3 levels compared to tau expression alone (Fig. 3).

Since CHIP decreases the level of cleaved caspase 3 when co-expressed with tau, we predicted that the level of caspase cleaved truncated tauC3 would also be decreased. Dolan et al. [20] showed that CHIP interacts more strongly with tauC3 than full length tau. Therefore, we co-transfected H4-cells with full length CHIP and $4 \mathrm{R}$-tau for $48 \mathrm{~h}$ and checked for the level of cleaved tau at $\mathrm{Asp}^{421}$. Indeed, the co-expression of CHIP caused a significant decrease tauC3 in vitro (Fig. 4). 

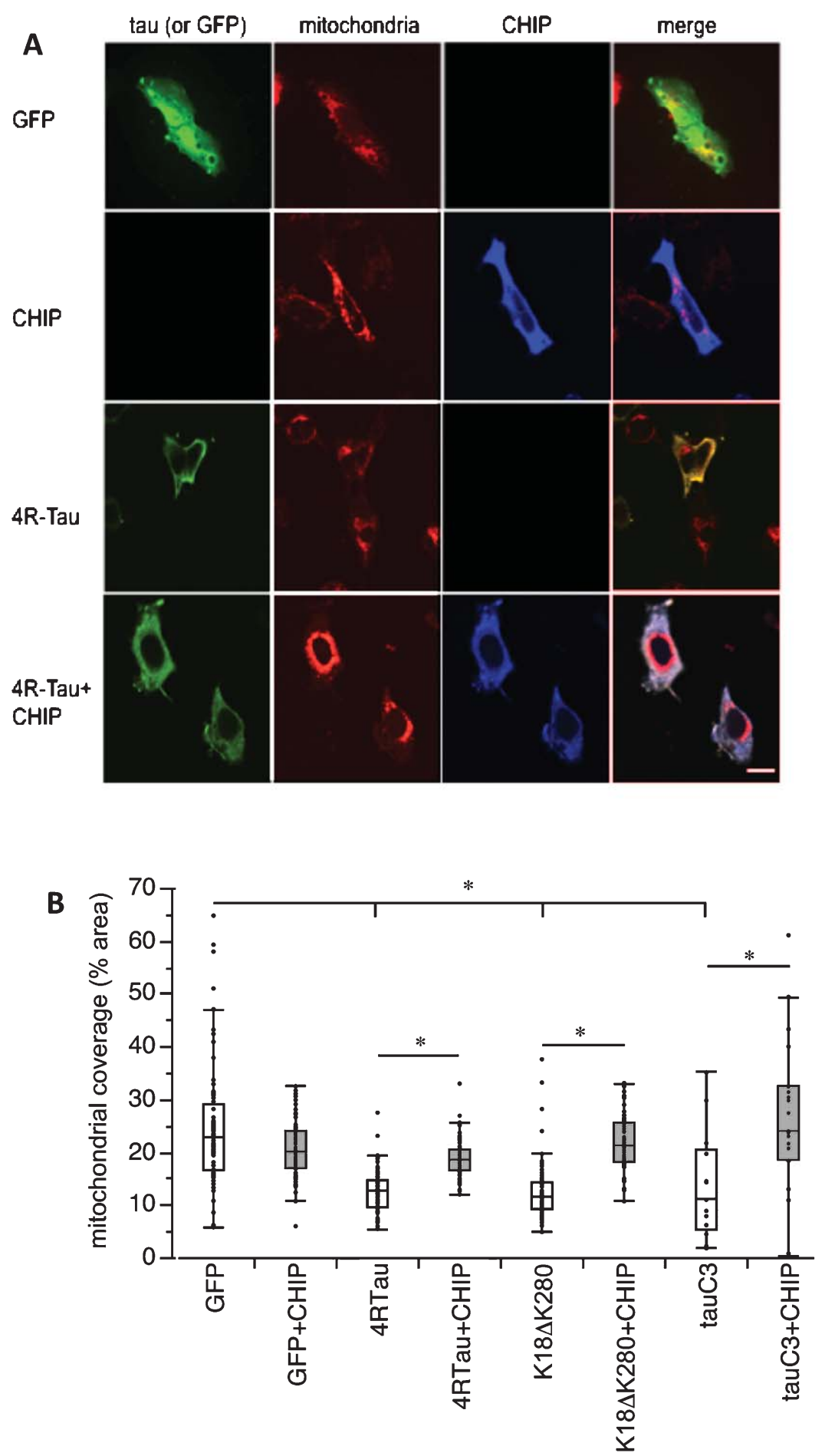

Fig. 5. Mitochondria transport deficits are rescued by CHIP co-expression after $24 \mathrm{~h}$. A) Representative photomicrographs showing H4 cells co-transfected with GFP-Tau (green) and either CHIP or an empty vector (GFP) to have an equimolar ratio of DNA in each condition. Mitochondria were labeled with Mitotracker Red (red). CHIP-myc expression was detected with an anti-myc antibody (blue). Scale bar $20 \mu m$. B) Quantification of mitochondrial distribution shows a significant effect of Tau on mitochondrial clustering occupied by overexpression of 4R-Tau, TauC3, or K18 $\mathrm{K} 280$ (12-14\% mito area in tau overexpressing cells compared to 20-24\% mito area in GFP or CHIP expressing cells; $p<0.0001$, Mann-Whitney test GFP control versus tau). CHIP co-expression led to a significant recovery in the area of mitochondria around the nucleus (19-26\% mito area). The data are non-parametric thus are presented as box plots. 
These results indicate that CHIP is involved in degradation of caspases and caspase-cleaved tau.

\section{Mitochondrial transport deficits are rescued by CHIP co-expression}

Alterations in tau are known to disrupt microtubule based transport including that of mitochondria. To determine whether CHIP rescues mitochondrial transport deficits, we cultured $\mathrm{H} 4$-cells and cotransfected them with CHIP and tau (4R-tau, tauC3, or $\mathrm{K} 18 \Delta \mathrm{K} 280$ ) for $24 \mathrm{~h}$ followed by fixation and immunocytochemistry and confocal imaging of mitochondria. As expected, we observed a significant impairment of mitochondrial distribution with overexpression of all three tau constructs $(p<0.0001$, Wilcoxon test for GFP control versus 4R-tau, GFP versus tauC3, and GFP versus K18 $\Delta$ K280) (Fig. 5). The percentage of the cytoplasm occupied by mitochondria in tau overexpressing cells was 12-14\% compared to $20-24 \%$ of the cytoplasm occupied by mitochondria in GFP or CHIP expressing cells. CHIP co-expression led to a significant recovery in the area of mitochondria around the nucleus (19-26\%). Although all tau constructs had a statistically significant effect on mitochondrial distribution within the cell body, there were no significant differences between the tau constructs in terms of the tau-induced change in mitochondrial localization in H4-cells (Wilcoxon test

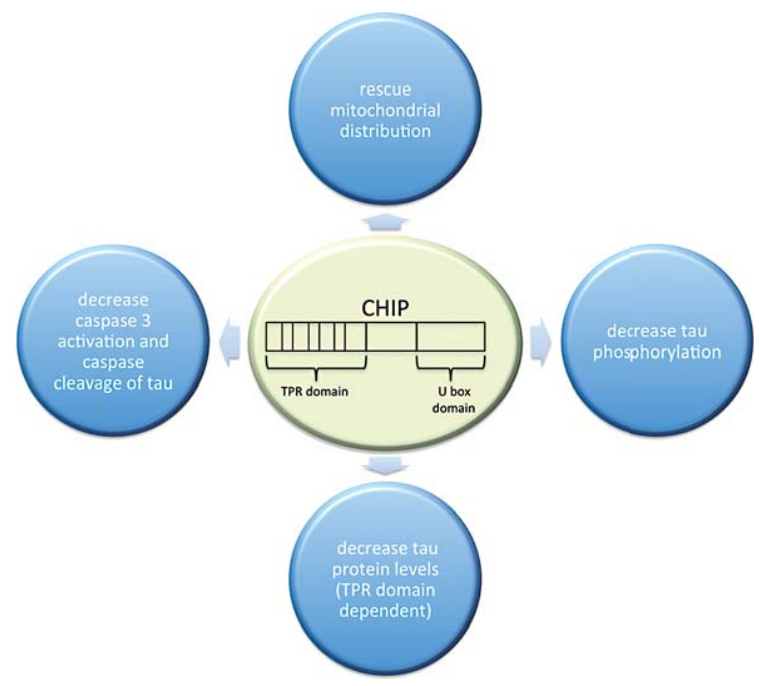

Fig. 6. Summary. Co-expression of CHIP with three different tau constructs in $\mathrm{H} 4$ cells leads to decreased tau protein levels, decreased tau phosphorylation, decreased caspase 3 activation and caspase cleavage of tau, and rescues mitochondrial distribution in cells. Together, these data indicate that increasing CHIP levels or activity could be beneficial in tauopathies. $p>0.05$ for all pairwise comparisons of tau expressing conditions). Taken together, these data indicate that tau-overexpression leads to abnormal mitochondrial trafficking that can be rescued by CHIP-co-expression.

\section{DISCUSSION}

Pathological changes in tau including aberrant phosphorylation, misfolding, mislocalization, and aggregation are tightly linked with neurodegeneration in AD and other tauopathies. Recent failures in amyloid directed therapeutics have rejuvenated the field of therapies aimed at reducing the pathological changes in tau [20]. Several studies indicate that reducing tau levels using active or passive immunotherapy is beneficial in mouse models of tauopathy [21-23]. Another promising therapeutic avenue is the use of chaperone pathways to reduce levels of toxic tau species. CHIP has been shown to reduce tau and phospho-tau levels in several studies $[15,16,24]$. In the present study, we expand on this data to show that the toxic effects of three different tau constructs: $4 \mathrm{R}$ tau, tauC3, and the aggregate prone $\mathrm{K} 18 \Delta \mathrm{K} 280$ fragment can all be rescued by CHIP overexpression in cell culture. We demonstrate that 1) CHIP reduces levels of tau protein, which requires the TPR-domain; 2) CHIP decreases phosphorylation and caspase cleavage of tau; and 3) mitochondrial distribution deficits induced by tau are rescued by CHIP co-expression.

CHIP has been shown to interact with tau and to ubiquitinate tau directly [25]. It also appears to work in concert with both Hsp70 [15] and Hsp90 [24] in degrading toxic tau species. Our data confirm that CHIP overexpression leads to tau degradation with three different tau constructs. While both the TPR domain and the U-box domain have been shown to be necessary for CHIP binding to tau, perhaps in a complex of proteins [15], our data indicate that only the Hsp70 binding TPR domain is necessary for reductions in tau levels with CHIP overexpression. Previous studies have shown an increase in Hsp70 levels in both human AD and JNPL3 mice [16]. In contrast, we do not observe a consistent upregulation of Hsp70 levels across the different types of tau examined, indicating that CHIP binding to Hsp70 is sufficient to reduce tau levels without increasing overall Hsp70 levels. The relationship between tau and Hsp70 has been extensively studied with conflicting results. Some groups find that inhibiting Hsp70 is beneficial for reducing tau abnormalities [26]; while others find that Hsp70 induction or overexpression reduce tau levels [15]. A recent 
study indicates that different variants in the Hsp70 family have different effects on tau clearance with Hsc70 (heat shock cognate 70) slowing tau clearance and Hsp72 accelerating tau clearance and recruiting CHIP binding [27], potentially explaining some of the conflicting data on Hsp70 levels. The full picture of the role of chaperones in tau pathology will be difficult to determine due to the complex interactions between different chaperones and their targets in different aggregation/folding states. For example, recent work has demonstrated that Hsp90 specifically selects for late folding intermediates of tau that likely remain available for binding after Hsp70 is bound to tau [28]. Understanding the complex relationships of chaperones in removing pathological proteins is important beyond tauopathies since protein misfolding and aggregation are common to many diseases. CHIP has also been shown to be effective in clearing pathological $\alpha$-synuclein [29], and in the 3x-Tg-AD mouse model, amyloid- $\beta$ accumulation is associated with decreasing CHIP levels and overexpressing CHIP reduces amyloid- $\beta$-associated tau phosphorylation [30].

Non-apoptotic caspase 3 activation has been implicated both in tau aggregation [9] and in synaptic degeneration in AD [31]. Previous work by Dickey and colleagues showed that deletion of CHIP leads to accumulation of phospho-tau and caspase 3 cleaved tau [17]. Here we confirm that CHIP overexpression reduces both caspase 3 activation and caspase cleavage of tau with 3 different tau isoforms.

Several studies indicate that tau disrupts transport of organelles in the cell [3, 6, 7, 32-34]. We have previously shown that overexpression of full-length tau and tauC3 in CHO-cells, H4-cells, and primary neuronal cultures change normal mitochondrial distribution within the cell body followed by mitochondrial clustering [4, 6, 7]. Similar effects tau impairing mitochondrial distribution occur in rTg4510 tau overexpressing mice and human $\mathrm{AD}$ brain [8]. Here we report the novel result that CHIP overexpression rescues mitochondrial distribution.

In summary, these data indicate that the upregulation of the co-chaperone CHIP can rescue pathological effects of tau and may be a promising therapeutic target.

\section{ACKNOWLEDGMENTS}

This work was funded by The Alzheimer's Association Zenith Award, Alzheimer's Research UK/Chief Scientist Office Scotland, German National Academic
Foundation, Foundation Family Klee Young Scientist Award, DZNE, Tau Consortium (USA).

Authors' disclosures available online (http://www. j-alz.com/disclosures/view.php?id=2581).

\section{SUPPLEMENTARY MATERIAL}

The supplementary material is available in the electronic version of this article: http://dx.doi.org/ 10.3233/JAD-142094.

\section{REFERENCES}

[1] Spires-Jones T, Stoothoff W, de Calignon A, Jones P, Hyman B (2009) Tau pathophysiology in neurodegeneration: A tangled issue. Trends Neurosci 32, 150-159.

[2] Mandelkow EM, Mandelkow E (2012) Biochemistry and cell biology of tau protein in neurofibrillary degeneration. Cold Spring Harb Perspect Med 2, a006247.

[3] Ebneth A, Godemann R, Stamer K, Illenberger S, Trinczek B, Mandelkow E (1998) Overexpression of tau protein inhibits kinesin-dependent trafficking of vesicles, mitochondria, and endoplasmic reticulum: Implications for Alzheimer's disease. J Cell Biol 143, 777-794.

[4] Mandelkow EM, Thies E, Trinczek B, Biernat J, Mandelkow E (2004) MARK/PAR1 kinase is a regulator of microtubuledependent transport in axons. J Cell Biol 167, 99-110.

[5] Dubey M, Chaudhury P, Kabiru H, Shea TB (2008) Tau inhibits anterograde axonal transport and perturbs stability in growing axonal neurites in part by displacing kinesin cargo: Neurofilaments attenuate tau-mediated neurite instability. Cell Motil Cytoskeleton 65, 89-99.

[6] Stoothoff W, Jones PB, Spires-Jones TL, Joyner D, Chhabra E, Bercury K, Fan Z, Xie H, Bacskai B, Edd J, Irimia D, Hyman BT (2009) Differential effect of three-repeat and fourrepeat tau on mitochondrial axonal transport. J Neurochem 111, 417-427.

[7] Thies E, Mandelkow EM (2007) Missorting of tau in neurons causes degeneration of synapses that can be rescued by the kinase MARK2/Par-1. J Neurosci 27, 2896-2907.

[8] Kopeikina KJ, Carlson GA, Pitstick R, Ludvigson AE, Peters A, Luebke JI, Koffie RM, Frosch MP, Hyman BT, SpiresJones TL (2011) Tau accumulation causes mitochondrial distribution deficits in neurons in a mouse model of tauopathy and in human Alzheimer's disease brain. Am J Pathol 179, 2071-2082.

[9] de Calignon A, Fox LM, Pitstick R, Carlson GA, Bacskai BJ, Spires-Jones TL, Hyman BT (2010) Caspase activation precedes and leads to tangles. Nature 464, 1201-1204.

[10] Kirkin V, McEwan DG, Novak I, Dikic I (2009) A role for ubiquitin in selective autophagy. Mol Cell 34, 259-269.

[11] Goldberg AL (2003) Protein degradation and protection against misfolded or damaged proteins. Nature 426, 895-899.

[12] Lee MJ, Lee JH, Rubinsztein DC (2013) Tau degradation: The ubiquitin-proteasome system versus the autophagy-lysosome system. Prog Neurobiol 105, 49-59.

[13] Wang Y, Mandelkow E (2012) Degradation of tau protein by autophagy and proteasomal pathways. Biochem Soc Trans $\mathbf{4 0}$, 644-652.

[14] Shimura H, Schwartz D, Gygi SP, Kosik KS (2004) CHIP-Hsc70 complex ubiquitinates phosphorylated tau and enhances cell survival. J Biol Chem 279, 4869-4876. 
[15] Petrucelli L, Dickson D, Kehoe K, Taylor J, Snyder H, Grover A, De Lucia M, McGowan E, Lewis J, Prihar G, Kim J, Dillmann WH, Browne SE, Hall A, Voellmy R, Tsuboi Y, Dawson TM, Wolozin B, Hardy J, Hutton M (2004) CHIP and Hsp70 regulate tau ubiquitination, degradation and aggregation. Hum Mol Genet 13, 703-714.

[16] Sahara N, Murayama M, Mizoroki T, Urushitani M, Imai Y, Takahashi R, Murata S, Tanaka K, Takashima A (2005) In vivo evidence of CHIP up-regulation attenuating tau aggregation. J Neurochem 94, 1254-1263.

[17] Dickey CA, Yue M, Lin WL, Dickson DW, Dunmore JH, Lee WC, Zehr C, West G, Cao S, Clark AM, Caldwell GA, Caldwell KA, Eckman C, Patterson C, Hutton M, Petrucelli L (2006) Deletion of the ubiquitin ligase CHIP leads to the accumulation, but not the aggregation, of both endogenous phospho- and caspase-3-cleaved tau species. J Neurosci $\mathbf{2 6}$, 6985-6996.

[18] Dolan PJ, Johnson GV (2010) A caspase cleaved form of tau is preferentially degraded through the autophagy pathway. $J$ Biol Chem 285, 21978-21987.

[19] von Bergen M, Barghorn S, Li L, Marx A, Biernat J, Mandelkow EM, Mandelkow E (2001) Mutations of tau protein in frontotemporal dementia promote aggregation of paired helical filaments by enhancing local beta-structure. J Biol Chem 276, 48165-48174.

[20] Karran E, Mercken M, De Strooper B (2011) The amyloid cascade hypothesis for Alzheimer's disease: An appraisal for the development of therapeutics. Nat Rev Drug Discov 10, 698-712.

[21] Yanamandra K, Kfoury N, Jiang H, Mahan TE, Ma S, Maloney SE, Wozniak DF, Diamond MI, Holtzman DM (2013) Anti-tau antibodies that block tau aggregate seeding in vitro markedly decrease pathology and improve cognition in vivo. Neuron 80, 402-414.

[22] Boutajangout A, Ingadottir J, Davies P, Sigurdsson EM (2011) Passive immunization targeting pathological phosphotau protein in a mouse model reduces functional decline and clears tau aggregates from the brain. J Neurochem 118, 658667.

[23] d'Abramo C, Acker CM, Jimenez HT, Davies P (2013) Tau passive immunotherapy in mutant P301L mice: Antibody affinity versus specificity. PLoS One 8, e62402.

[24] Dickey CA, Kamal A, Lundgren K, Klosak N, Bailey RM, Dunmore J, Ash P, Shoraka S, Zlatkovic J, Eckman CB, Patterson C, Dickson DW, Nahman NS Jr, Hutton M, Burrows F, Petrucelli L (2007) The high-affinity HSP90-CHIP complex recognizes and selectively degrades phosphorylated tau client proteins. J Clin Invest 117, 648-658.

[25] Hatakeyama S, Matsumoto M, Kamura T, Murayama M, Chui DH, Planel E, Takahashi R, Nakayama KI, Takashima A (2004) U-box protein carboxyl terminus of Hsc70-interacting protein (CHIP) mediates poly-ubiquitylation preferentially on four-repeat Tau and is involved in neurodegeneration of tauopathy. J Neurochem 91, 299-307.

[26] Miyata Y, Li X, Lee H-FF, Jinwal UK, Srinivasan SR, Seguin SP, Young ZT, Brodsky JL, Dickey CA, Sun D, Gestwicki JE (2013) Synthesis and initial evaluation of YM-08, a bloodbrain barrier permeable derivative of the heat shock protein 70 (Hsp70) inhibitor MKT-077, which reduces tau levels. ACS Chem Neurosci 4, 930-939.

[27] Jinwal U, Akoury E, Abisambra J, O'Leary J, Thompson A, Blair L, Jin Y, Bacon J, Nordhues B, Cockman M, Zhang J, Li P, Zhang B, Borysov S, Uversky V, Biernat J, Mandelkow E, Gestwicki J, Zweckstetter M, Dickey C (2013) Imbalance of Hsp70 family variants fosters tau accumulation. FASEB $J$ 27, 1450-1459.

[28] Karagöz GE, Duarte AM, Akoury E, Ippel H, Biernat J, Morán Luengo T, Radli M, Didenko T, Nordhues BA, Veprintsev DB, Dickey CA, Mandelkow E, Zweckstetter M, Boelens R, Madl T, Rüdiger SG (2014) Hsp90-Tau complex reveals molecular basis for specificity in chaperone action. Cell $\mathbf{1 5 6}$, 963-974.

[29] Dimant H, Zhu L, Kibuuka LN, Fan Z, Hyman BT, McLean PJ (2014) Direct visualization of CHIP-mediated degradation of alpha-synuclein in vivo: Implications for PD therapeutics. PLoS One 9, e92098.

[30] Oddo S, Caccamo A, Tseng B, Cheng D, Vasilevko V, Cribbs DH, LaFerla FM (2008) Blocking Abeta42 accumulation delays the onset and progression of tau pathology via the $\mathrm{C}$ terminus of heat shock protein70-interacting protein: A mechanistic link between Abeta and tau pathology. J Neurosci 28, 12163-12175.

[31] Tai HC, Serrano-Pozo A, Hashimoto T, Frosch MP, SpiresJones TL, Hyman BT (2012) The synaptic accumulation of hyperphosphorylated tau oligomers in Alzheimer disease is associated with dysfunction of the ubiquitin-proteasome system. Am J Pathol 181, 1426-1435.

[32] Trinczek B, Ebneth A, Mandelkow EM, Mandelkow E (1999) Tau regulates the attachment/detachment but not the speed of motors in microtubule-dependent transport of single vesicles and organelles. J Cell Sci 112(Pt 14), 2355-2367.

[33] Seitz A, Kojima H, Oiwa K, Mandelkow EM, Song YH, Mandelkow E (2002) Single-molecule investigation of the interference between kinesin, tau and MAP2c. EMBO J 21, 4896-4905.

[34] Stamer K, Vogel R, Thies E, Mandelkow E, Mandelkow EM (2002) Tau blocks traffic of organelles, neurofilaments, and APP vesicles in neurons and enhances oxidative stress. J Cell Biol 156, 1051-1063. 